\title{
Thin-Film-Based Phase Plates for Transmission Electron Microscopy Fabricated From Metallic Glasses
}

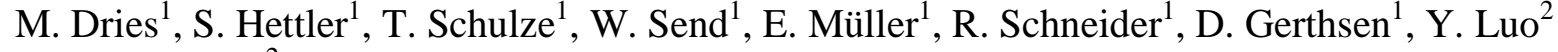 \\ and K. Samwer ${ }^{2}$ \\ ${ }^{1 .}$ Laboratorium für Elektronenmikroskopie, Karlsruher Institut für Technologie, Karlsruhe, Germany \\ 2. I. Physikalisches Institut, Universität Göttingen, Göttingen, Germany
}

Intense development of physical phase plates (PPs) in the past decade led to substantial improvements in transmission electron microscopy (TEM) imaging of weak-phase objects. Research has focused on thinfilm PPs, which are typically fabricated from amorphous carbon (aC)-films [1]. Amorphous carbon has two important properties, which are essential for phase-contrast TEM: A sufficiently high electrical conductivity and an amorphous structure to avoid Bragg diffraction in the PP material. Thin-film PPs based on aC-films have already become widely accepted to enhance the contrast of weak-phase objects in TEM [2]. However, the irradiation with high-energy electrons initiates a steady, irreversible degeneration of the aC-film, which reduces the lifetime of aC-film-based PPs. Therefore, recent investigations have focused on the search of alternative materials with an improved material stability $[3,4]$.

This study, for the first time, presents thin-film PPs fabricated from a metallic glass alloy. Metallic glasses are characterized by a high electrical conductivity and an amorphous structure. Moreover, structural degradation under the intense electron-beam is not expected if the crystallization temperature is high enough. $\mathrm{Zr}_{65.0} \mathrm{Al}_{7.5} \mathrm{Cu}_{27.5}$ (ZAC) was chosen for its favorable properties and its high crystallization temperature of $437^{\circ} \mathrm{C}$ [5]. We have applied Hilbert PPs (HPPs) in this work, which consist of a microstructured thin film located in the back focal plane of the objective lens [6]. The film thickness is adjusted in such a way, that a phase shift of $\pi$ is imposed on the electrons in one half of the diffraction pattern except for the zero-order beam. This yields an overall phase shift of $\pi / 2$ for spatial frequencies above the cut-on frequency.

The ZAC-film was sputtered on a cleaved mica-substrate and floated on a $\mathrm{Cu}$-grid. Using a focused ionbeam system, rectangular windows were structured into the ZAC-film, which yields HPPs in several meshes of the $\mathrm{Cu}$-grid. The $\mathrm{Cu}$-grid was mounted in an objective aperture stripe and implemented in the back focal plane of a Philips CM200 FEG/ST. At an acceleration voltage of $200 \mathrm{kV}$, the ZAC-film of $24 \mathrm{~nm}$ thickness induces a phase shift close to $\pi$.

Fig. 1a shows a cross-section TEM image of a ZAC-film sputtered on a Si-substrate. Even short periods at ambient air lead to an oxide layer of $4 \mathrm{~nm}$ thickness, which appears with intermediate gray contrast in Fig. 1a. The oxygen is also visible in the composition profile shown in Fig. 1b, which was obtained by energy dispersive X-ray spectroscopy (EDXS). The electrically insulating oxide layer causes electrostatic charging of the ZAC-film, which affects its phase shifting behavior. Therefore, a thin aC-coating was applied to the ZAC-film. Fig. 2a depicts the power spectrum of an amorphous test object, which demonstrates the desired phase shifting properties. The power spectrum is subdivided in a central stripe (red) and outer areas (green) with Thon-rings shifted by $\pi / 2$. The complementary behavior in the two regions is also demonstrated by the azimuthally averaged intensity profiles shown in Fig. 2 b.

Although amorphous carbon was not fully removed from the PP-production process, the properties of 
metallic glasses are promising to improve the applicability of thin-film PPs for phase-contrast TEM [7].

References:

[1] R Danev and K Nagayama, Ultramicroscopy 88 (2001), p. 243-252.

[2] R Danev et al, PNAS 111 (2014), p. 15635-15640.

[3] M Marko et al, J. Struct. Biol. 184 (2013), p. 237-244.

[4] M Dries et al, Ultramicroscopy 139 (2014), p. 29-37.

[5] R Rambousky, M Moske and K Samwer, Z. Phys. B 99 (1996), p. 387-391.

[6] R Danev and K Nagayama, J. Phys. Soc. Jpn. 73 (2004), p. 2718-2724.

[7] Financial support by the Deutsche Forschungsgemeinschaft (DFG).
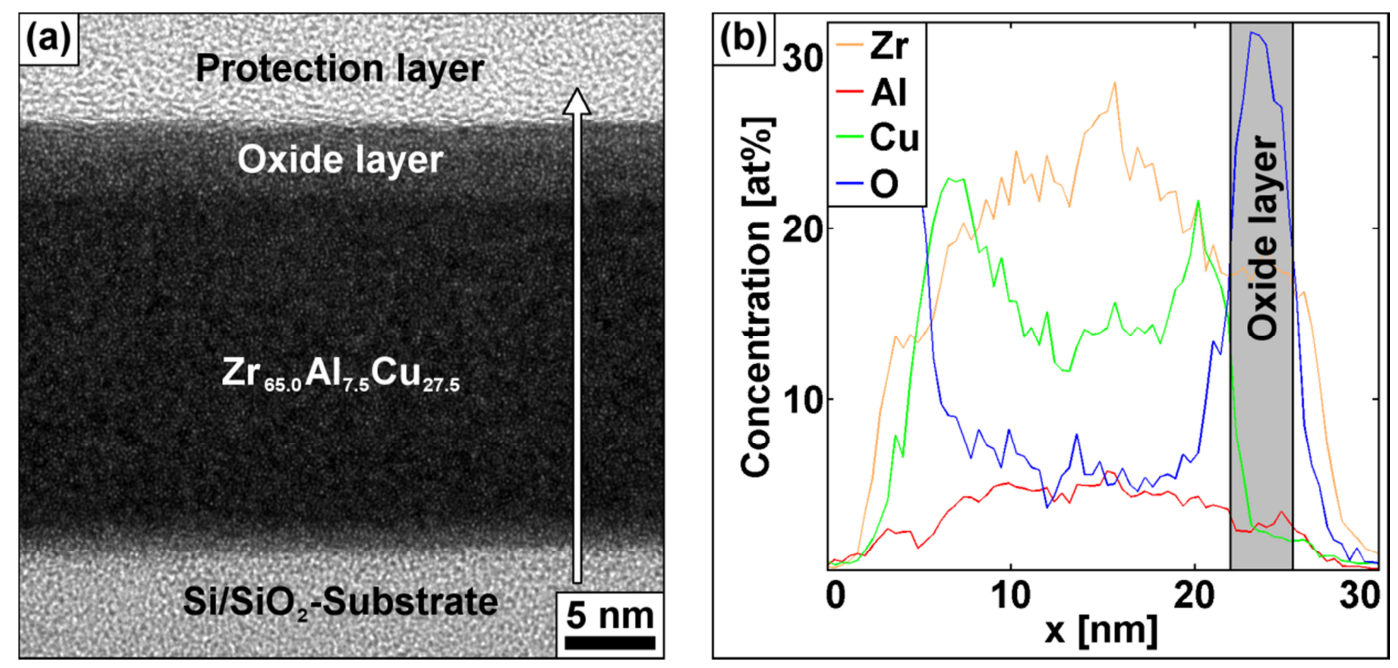

Figure 1. Formation of oxide layers at the surface of ZAC-films. (a) Cross-section TEM image of an oxidized ZAC-film with a Pt/C-protection layer on top. (b) Composition profile along the white arrow in (a) obtained by EDXS measurements.
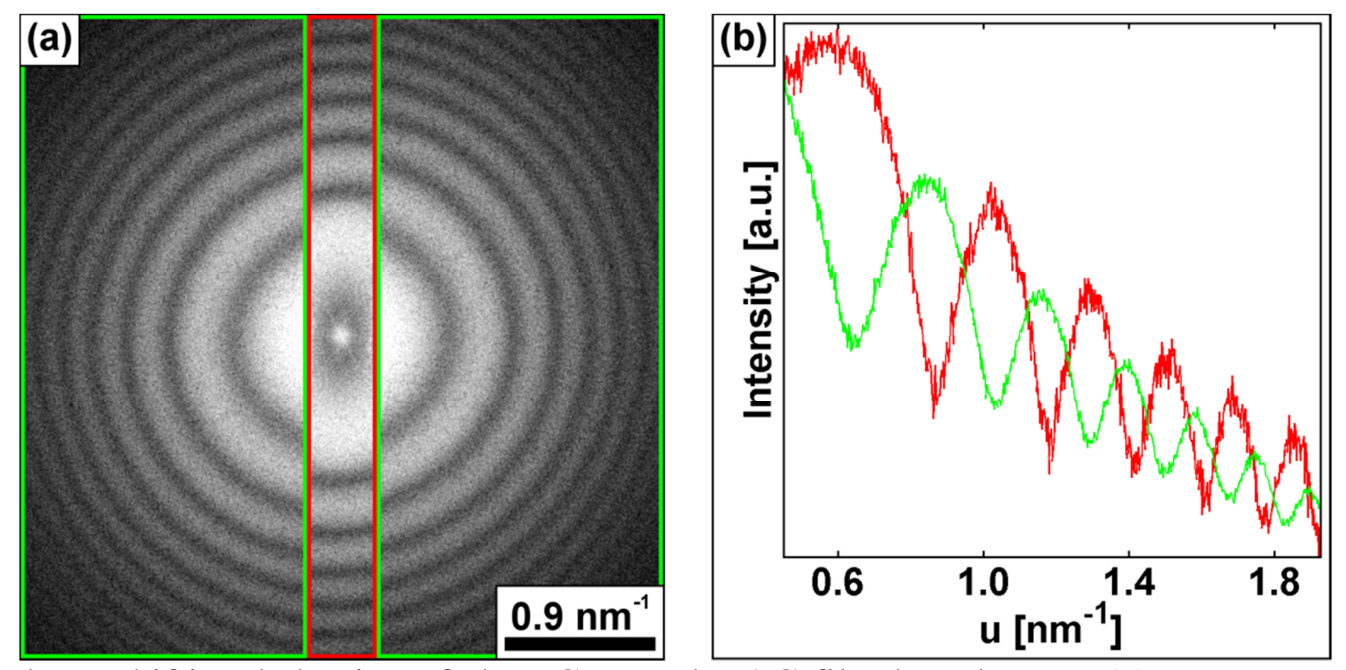

Figure 2. Phase shifting behavior of the aC-coated ZAC-film-based HPP. (a) Power spectrum of a phase-contrast TEM image of an amorphous test object. (b) Azimuthally averaged intensity profiles taken from the Thon-rings in the red and green regions of (a). 